patients have a higher rate of heart failure than non-Hispanic whites. Current guidelines recommend that proper screening tools must be used to identify and manage major depression disorder in HF patients, however many of these patients go unrecognized in the medical setting. The prevalence, management, and outcomes of depression among PuertoRican Hispanics living with heart failure is unknown. Objective: The purpose of this study is to evaluate the relationship between heart failure and depression in Hispanics with heart failure METHODS/STUDY POPULATION: To this end, we will perform a secondary analysis of data from the PR CardiovascularSurveillance Study (PRCSS). We will extract personal data from 4,461 medical records of patients admitted with heart failure (ICD-9 Codes 428) at 21 hospitals in Puerto Rico, during the years 2007, 2009 and 2011. For statistical methods, we will implement chi-square and t-tests at a significance level of 0.05 RESULTS/ANTICIPATED RESULTS: We expect to see older aged women with higher NYHA and pro-BNP levels to be associatedwith diagnosis of major depression disorder and worse in-hospital outcomes DISCUSSION/SIGNIFICANCE OF IMPACT: With this study, we would like to raise awareness about depression in patients with heart failure, and its role in improving patient outcomes. Moreover, we would like to determine if there are gender-specific health disparities among Puerto Rican Hispanics with heart failure

4200

Assessment of differential access to patient online portal (POP) by socioeconomic status (SES) and its impact on asthma care and research

Young J Juhn ${ }^{1}$, Chung-il Wi, Euijung Ryu, Sunghwan Sohn, Miguel Park, Joy Fladager Muth, Hee Yun Seol, Katherine King, and Hongfang Liu

${ }^{1}$ Mayo Clinic

OBJECTIVES/GOALS: Patient online portal (POP) allows patients to access electronic health records (EHRs) and have efficient communication with their clinicians. We assessed disparities in access to POP by families with different SES and its impact on asthma research which is little known in the literature. METHODS/ STUDY POPULATION: A randomized controlled trial testing the efficacy of an EHRs-based clinical decision support (CDS) system was conducted at a pediatric primary care setting of Mayo Clinic. Asthma Control Test (ACT) questionnaire was administered to parents every 3 months through phone or email for this study after consenting, and reminders were sent to unanswered subjects through the POP. SES was measured by HOUSES (in quartiles), a validated individual-level SES index based on housing features (the higher HOUSES, the higher SES).The association of HOUSES with availability of POP access and missing ACT score rate was assessed. RESULTS/ANTICIPATED RESULTS: The mean age of 184 participants was 9.0 years (57\% male) and parents of 152 (83\%) children had POP. Only $68 \%$ of children from lowest HOUSES (Q1) had access to POP (vs. 74\% (Q2), 88\% (Q3), and 92\% (Q4; highest SES); $\mathrm{p}=.02)$. ACT score was completed by $144(78 \%), 150$ (82\%), 171 (94\%), and $164(95 \%)$ at each intervention conducted every 3 months with a total of 61 (33\%) missing at least once. Overall, children whose parents had access to POP had a lower missing rate in ACT score at all interventions during the study; $16 \%$ (those with access to POP) vs. $47 \%$ (those without), $13 \%$ vs. $44 \%$, $3 \%$ vs. $16 \%$, and $1 \%$ vs. $23 \%$ for $1^{\text {st }}, 2^{\text {nd }}, 3^{\text {rd }}$, and $4^{\text {th }}$ intervention, respectively $(\mathrm{p}<.007$ for all). DISCUSSION/SIGNIFICANCE OF IMPACT: There are significant disparities in access to POP by
SES defined by HOUSES which impact availability of ACT score resulting in a systematic bias in asthma research and potentially widening disparities in asthma care. CONFLICT OF INTEREST DESCRIPTION: NA.

4537

\section{Association between Geographic Socioeconomic Disadvantage and Incidence of Total Hip Replacement Surgery}

Rafa Rahman ${ }^{1}$, Joseph K. Canner ${ }^{1}$, Elliot R. Haut, MD, $\mathrm{PhD}^{1}$, and Casey J. Humbyrd ${ }^{1}$

${ }^{1}$ Johns Hopkins University School of Medicine

OBJECTIVES/GOALS: Total hip replacement (THR) improves function for those with arthritis, but not all patients have equal access to this elective procedure. To better geographically target healthcare resources, we explored whether geographic socioeconomic disadvantage is associated with incidence of elective THR. METHODS/ STUDY POPULATION: We performed a cross-sectional analysis of data in the state of Maryland from 2013-2019. We categorized 5 -digit zipcodes into national quartiles of socioeconomic disadvantage using the Area Deprivation Index (ADI). For each zipcode, we calculated the THR incidence rate using Maryland Health Services Cost Review Commission (HSCRC) inpatient and outpatient data in those age 65 years and older. We included only elective THRs. We analyzed the association between a zipcode's disadvantage quartile and THR incidence rate using multivariate linear regression, correcting for differences across zipcodes in gender, race, and ethnicity distributions, and distance to the nearest hospital performing THRs. RESULTS/ANTICIPATED RESULTS: We analyzed 414 zipcodes with overall average THR rate of 370.8 per 100,000 persons $>65$ yo per year. Relative to zipcodes in the least socioeconomically disadvantaged quartile, those in the second-least disadvantaged had 82.2 fewer THRs per 100,000 persons $>65$ yo per year, those in the second-most disadvantaged had 144.2 fewer, and those in the most disadvantaged had 207.4 fewer (all p65yo per year, those in the second-most disadvantaged had 136.2 fewer, and those in the most disadvantaged had 182.9 fewer (all $\mathrm{p}<.05$ ). DISCUSSION/ SIGNIFICANCE OF IMPACT: More socioeconomically disadvantaged areas have significantly lower rates of elective THR, independent of differences in demographics and hospital proximity. These findings show how disparities can affect access and outcomes, and should inform targeting of community-level education and intervention.

4070

Association of Interpersonal Processes of Care and Health Outcomes in Patients with Type II Diabetes Hadley Reid ${ }^{1}$, Olivia M Lin ${ }^{1}$, Rebecca L Fabbro ${ }^{1}$, Kimberly S Johnson ${ }^{1}$, Laura P. Svetkey, MD ${ }^{1}$, and Bryan C Batch ${ }^{1}$

${ }^{1}$ Duke University

OBJECTIVES/GOALS: 1 . Understand the association between patient perceptions of care measured by the Interpersonal Processes of Care (IPC) Survey and glycemic control, appointment no-shows/cancellations and medication adherence in patients with type II diabetes. 2. Determine how these relationships differ by race for non-Hispanic White and Black patients. METHODS/STUDY POPULATION: This is a cross-sectional study of a random sample of 100 White and 100 Black Type II diabetic patients followed in 